\title{
EXISTENCE OF SOLUTIONS AND CONTROLLABILITY OF NONLINEAR INTEGRODIFFERENTIAL SYSTEMS IN BANACH SPACES
}

\author{
K. BALACHANDRAN AND J. Y. PARK
}

Received 17 January 2002

We prove the existence of mild and strong solutions of integrodifferential equations with nonlocal conditions in Banach spaces. Further sufficient conditions for the controllability of integrodifferential systems are established. The results are obtained by using the Schauder fixed-point theorem. Examples are provided to illustrate the theory.

\section{Introduction}

Byszewski [9] has studied the existence and uniqueness of mild, strong, and classical solutions of the following nonlocal Cauchy problem:

$$
\begin{gathered}
\frac{d u(t)}{d t}+A u(t)=f(t, u(t)), \quad t \in(0, a], \\
u\left(t_{0}\right)+g\left(t_{1}, t_{2}, \ldots, t_{p}, u(\cdot)\right)=u_{0},
\end{gathered}
$$

where $0 \leq t_{0}<t_{1}<\cdots<t_{p} \leq a, a>0,-A$ is the infinitesimal generator of a $C_{0}$-semigroup in a Banach space $X, u_{0} \in X$, and $f:[0, a] \times X \rightarrow X, g:[0, a]^{p} \times X \rightarrow X$ are given functions. Subsequently he has investigated the same type of problem for different kinds of evolution equations in Banach spaces $[10,11,12,13,14]$. Ntouyas and Tsamatos [31] have established the global existence of solutions of semilinear evolution equations with nonlocal conditions. Balachandran [1], Balachandran and Ilamaran [6], Balachandran and Chandrasekaran [3], Dauer and Balachandran [17], and Balachandran et al. [7] have studied the nonlocal Cauchy problem for various classes of integrodifferential equations. Physical motivation for this kind of problem is given in $[18,25]$.

It is well known [36] (when $A=0$ and $g=0$ ) that only the continuity of $f$ is not sufficient to assure local existence of solutions, even when $X$ is a Hilbert space. Therefore, one has to restrict either the function $f$ or the semigroup operator. Usually restrictions on $f$ are imposed, as either $f$ should satisfy the local Lipschitz condition, or be monotonic, or be completely continuous. Here we assume that the nonlinear terms satisfy the boundedness condition. 


\section{Preliminaries}

Consider the one-dimensional model in viscoelasticity

$$
\begin{gathered}
\rho u_{t t}(t, x)+k u_{t}(t, x)=\psi_{x}(t, x)+h(t, x), \\
\psi(t, x)=E u_{x}(t, x)+\int_{0}^{t} a(t-s) u_{x}(s, x) d s, \quad(t, x) \in \mathbb{R}^{+} \times[0,1], \\
u(t, 0)=u(t, 1)=0, \quad t \in \mathbb{R}^{+}, \\
u(0, x)=u_{0}(x), \quad x \in[0,1],
\end{gathered}
$$

where $u$ is the displacement, $\psi$ is the stress, $h$ is the external force, $\rho, E>0$ and $k$ are constants, and $\mathbb{R}^{+}=[0, \infty)$. The first equation is the linear momentum equation while the second is the constitutive relation between stress and strain. If we set $E=1, w=u_{t}$, and $v=u_{x}$, then we obtain

$$
\begin{aligned}
{\left[\begin{array}{c}
v^{\prime}(t) \\
w^{\prime}(t)
\end{array}\right]=} & {\left[\begin{array}{cc}
0 & \partial_{x} \\
\frac{\partial_{x}}{\rho} & 0
\end{array}\right]\left\{\left[\begin{array}{l}
v(t) \\
w(t)
\end{array}\right]+\int_{0}^{t}\left[\begin{array}{cc}
a(t-s) & 0 \\
0 & 0
\end{array}\right]\left[\begin{array}{c}
v(t) \\
w(t)
\end{array}\right] d s\right\} } \\
& +\left[\begin{array}{cc}
0 & 0 \\
0 & -\frac{k}{\rho}
\end{array}\right]\left[\begin{array}{c}
v(t) \\
w(t)
\end{array}\right]+\left[\begin{array}{c}
0 \\
\frac{h(t)}{\rho}
\end{array}\right], \quad t \geq 0 .
\end{aligned}
$$

The above equation can be written in the abstract form as

$$
\begin{gathered}
x^{\prime}(t)=A\left[x(t)+\int_{0}^{t} F(t-s) x(s)\right] d s+K x(t)+f(t), \quad t \geq 0, \\
x(0)=x_{0} .
\end{gathered}
$$

Here $A$ is an unbounded operator, while $K$ and $F(t)$ are bounded operators for $t \geq 0$ on a Banach space $X$.

In general, $F(t) A \neq A F(t)$ and $A(x+y)=A x+A y$ is true only if $x, y \in D(A)$. Thus the above equation is distinct from the equation

$$
\begin{gathered}
x^{\prime}(t)=A x(t)+\int_{0}^{t} F(t-s) A x(s) d s+K x(t)+f(t), \quad t \geq 0, \\
x(0)=x_{0},
\end{gathered}
$$

which has been studied in [19]. If we consider a nonlinear term instead of $K x(t)+f(t)$, then (2.4) becomes

$$
\begin{gathered}
x^{\prime}(t)=A\left[x(t)+\int_{0}^{t} F(t-s) x(s)\right] d s+f(t, x(t)), \quad t \geq 0, \\
x(0)=x_{0} .
\end{gathered}
$$

This type of equation also occurs in the study of viscoelastic beams and thermoviscoelasticity (see $[19,29]$ and the references therein). If the nonlocal condition (1.2) is 
introduced to the above equation, it will also have a better effect than the classical condition. The nonlocal Cauchy problem for the above equation has been discussed by Lin and Liu [28]. The Cauchy problem for integrodifferential equations in Banach spaces has been studied by several authors [16, 21, 23, 24].

In this section we consider the following integrodifferential equation with a nonlocal condition:

$$
\begin{aligned}
& x^{\prime}(t)= A\left[x(t)+\int_{0}^{t} F(t-s) x(s) d s\right]+f(t, x(t)) \\
&+ \int_{0}^{t} g\left(t, s, x(s), \int_{0}^{s} k(s, \tau, x(\tau)) d \tau\right) d s, \quad t \in[0, a]=J, \\
& x(0)+h\left(x\left(t_{1}\right), \ldots, x\left(t_{p}\right)\right)=x_{0},
\end{aligned}
$$

where $A$ generates a strongly continuous semigroup in a Banach space $X, F(t)$ is a bounded operator for $t \in J$, and $f: J \times X \rightarrow X, k: \Delta \times X \rightarrow X, g: \Delta \times X \times X \rightarrow X$, and $h: X^{p} \rightarrow$ $X$ are given functions. Here $\Delta=\{(t, s): 0 \leq s \leq t \leq a\}, F(t) \in B(X), t \in J, F(t): Y \rightarrow Y$, and for $x(\cdot)$ continuous in $Y, A F(\cdot) x(\cdot) \in L^{1}(J, X)$. For $x \in X, F^{\prime}(t) x$ is continuous in $t \in J$, where $B(X)$ is the space of all linear and bounded operators on $X$, and $Y$ is the Banach space formed from $D(A)$, the domain of $A$, endowed with the graph norm. Then there exists a unique resolvent operator for the equation

$$
x^{\prime}(t)=A\left[x(t)+\int_{0}^{t} F(t-s) x(s) d s\right]
$$

The resolvent operator $R(t) \in B(X)$ for $t \in J$ satisfies the following conditions [20]:

(a) $R(0)=I$ (the identity operator on $X$ ),

(b) for all $x \in X, R(t) x$ is continuous for $t \in J$,

(c) $R(t) \in B(Y), t \in J$. For $y \in Y, R(t) y \in C^{1}([0, a], X) \cap C([0, a], Y)$ and

$$
\begin{aligned}
\frac{d}{d t} R(t) y & =A\left[R(t) y+\int_{0}^{t} F(t-s) R(s) y d s\right] \\
& =R(t) A y+\int_{0}^{t} R(t-s) A F(s) y d s, \quad t \in J .
\end{aligned}
$$

The existence and uniqueness of solutions via variations of constants formula and other properties of resolvent operators have been studied in [20]. In this paper we study the existence of a mild and strong solution of the integrodifferential equation (2.6a) with nonlocal condition (2.6b) by utilizing the techniques developed by Pazy [33], Byszewski [9], and Lin and Liu [28]. The results generalize the results of [14,28]. We also study the controllability problem for the above equation.

Let $Y=C(J, X)$ and define the sets

$$
X_{r}=\{x \in X:\|x\| \leq r\}, \quad Y_{r}=\{y \in Y:\|y\| \leq r\},
$$

where the constant $r$ is defined below. Assume the following conditions. 
Existence of solutions and controllability

(i) The resolvent operator $R(t)$ is compact and there exists a constant $M_{1}>0$ such that

$$
\|R(t)\| \leq M_{1}
$$

(ii) The nonlinear operators $f: J \times X \rightarrow X, g: \Delta \times X \times X \rightarrow X$, and $k: \Delta \times X \rightarrow X$ are continuous and there exist constants $M_{2}>0, M_{3}>0$ such that

$$
\begin{array}{rlrl}
\|f(t, x(t))\| & \leq M_{2} & & \text { for } t \in J, x \in X_{r}, \\
\|g(t, s, x(s), y(s))\| \leq M_{3} & & \text { for }(t, s) \in \Delta, x, y \in X_{r} .
\end{array}
$$

(iii) The operator $h: I^{p} \times X \rightarrow X$ is continuous and there exists a constant $H>0$ such that

$$
\begin{gathered}
\left\|h\left(x\left(t_{1}\right), \ldots, x\left(t_{p}\right)\right)\right\| \leq H \quad \text { for } x \in Y_{r}, \\
h\left(\alpha x\left(t_{1}\right)+(1-\alpha) y\left(t_{1}\right), \ldots, \alpha x\left(t_{p}\right)+(1-\alpha) y\left(t_{p}\right)\right) \\
=\alpha h\left(x\left(t_{1}\right), \ldots, x\left(t_{p}\right)\right)+(1-\alpha) h\left(y\left(t_{1}\right), \ldots, y\left(t_{p}\right)\right) \quad \text { for } x, y \in Y_{r} .
\end{gathered}
$$

(iv) The set $\left\{y(0): y \in Y_{r}, y(0)=x_{0}-h\left(y\left(t_{1}\right), \ldots, y\left(t_{p}\right)\right)\right\}$ is precompact in $X$.

To simplify the notation, we take

$$
Q(t)=\int_{0}^{t} k(t, s, x(s)) d s
$$

Then (2.6a) takes the form

$$
\begin{gathered}
x^{\prime}(t)=A\left[x(t)+\int_{0}^{t} F(t-s) x(s) d s\right]+f(t, x(t))+\int_{0}^{t} g(t, s, x(s), Q(s)) d s, \\
x(0)+h\left(x\left(t_{1}\right), \ldots, x\left(t_{p}\right)\right)=x_{0} .
\end{gathered}
$$

Definition 2.1. A continuous solution $x(t)$ of the integral equation

$$
\begin{aligned}
x(t)= & R(t)\left[x_{0}-h\left(x\left(t_{1}\right), \ldots, x\left(t_{p}\right)\right)\right] \\
& +\int_{0}^{t} R(t-s)\left[f(s, x(s))+\int_{0}^{s} g(s, \tau, x(\tau), Q(\tau)) d \tau\right] d s
\end{aligned}
$$

is called a mild solution of problem (2.6).

Definition 2.2. A function $x$ is said to be a strong solution of problem (2.6) on $J$ if $x$ is differentiable a.e. on $J, x^{\prime} \in L^{1}(J, X)$ and satisfies

$$
\begin{gathered}
x^{\prime}(t)=A\left[x(t)+\int_{0}^{t} F(t-s) x(s) d s\right]+f(t, x(t))+\int_{0}^{t} g(t, s, x(s), Q(s)) d s, \\
x(0)+h\left(x\left(t_{1}\right), \ldots, x\left(t_{p}\right)\right)=x_{0} \quad \text { a.e. on } J .
\end{gathered}
$$




\section{Existence of mild and strong solutions}

Theorem 3.1. Assume that hypotheses (i), (ii), (iii), and (iv) hold. Then problem (2.6) has a mild solution on $\mathrm{J}$.

Proof. We define the set $Y_{0}$ in $Y$ by

$$
Y_{0}=\left\{x \in Y: x(0)+h\left(x\left(t_{1}\right), \ldots, x\left(t_{p}\right)\right)=x_{0},\|x(t)\| \leq r \text { for } 0 \leq t \leq a\right\}
$$

where $r=M_{1}\left\|x_{0}\right\|+H M_{1}+M_{1} a\left(M_{2}+M_{3} a\right)$.

Clearly, $Y_{0}$ is a bounded closed convex subset of $Y$.

Define a mapping $\Psi: Y \rightarrow Y_{0}$ by

$$
\begin{aligned}
(\Psi x)(t)= & R(t)\left[x_{0}-h\left(x\left(t_{1}\right), \ldots, x\left(t_{p}\right)\right)\right] \\
& +\int_{0}^{t} R(t-s)\left[f(s, x(s))+\int_{0}^{s} g(s, \tau, x(\tau), Q(\tau)) d \tau\right] d s .
\end{aligned}
$$

Since

$$
\begin{aligned}
\|(\Psi x)(t)\| \leq & \left\|R(t) x_{0}\right\|+\left\|R(t) h\left(x\left(t_{1}\right), \ldots, x\left(t_{p}\right)\right)\right\| \\
& +\int_{0}^{t}\left\|R(t-s)\left[f(s, x(s))+\int_{0}^{s} g(s, \tau, x(\tau), Q(\tau)) d \tau\right]\right\| d s \\
\leq & M_{1}\left\|x_{0}\right\|+H M_{1}+M_{1} a\left(M_{2}+M_{3} a\right)=r,
\end{aligned}
$$

then $\Psi$ maps $Y_{0}$ into $Y_{0}$. Further, the continuity of $\Psi$ from $Y_{0}$ into $Y_{0}$ follows from the fact that $f, g, k$, and $h$ are continuous. Moreover $\Psi$ maps $Y_{0}$ into a precompact subset of $Y_{0}$. We prove that the set $Y_{0}(t)=\left\{(\Psi x)(t): x \in Y_{0}\right\}$ is precompact in $X$, for every fixed $t, 0 \leq t \leq a$. For $t=0$, the set $Y_{0}(0)$ is precompact in $X$. Let $t>0$ be fixed. Define, for $0<\epsilon<t$,

$$
\begin{aligned}
\left(\Psi_{\epsilon} x\right)(t)= & R(t) x_{0}-R(t) h\left(x\left(t_{1}\right), \ldots, x\left(t_{p}\right)\right) \\
& +\int_{0}^{t-\epsilon} R(t-s)\left[f(s, x(s))+\int_{0}^{s} g(s, \tau, x(\tau), Q(\tau)) d \tau\right] d s .
\end{aligned}
$$

Since $R(t)$ is compact for every $t>0$, the set

$$
Y_{\epsilon}(t)=\left\{\left(\Psi_{\epsilon} x\right)(t): x \in Y_{0}\right\}
$$

is precompact in $X$ for every $\epsilon, 0<\epsilon<t$.

Further, for $x \in Y_{0}$, we have

$$
\begin{aligned}
& \left\|(\Psi x)(t)-\left(\Psi_{\epsilon} x\right)(t)\right\| \\
& \leq\left\|\int_{t-\epsilon}^{t} R(t-s)\left[f(s, u(s))+\int_{0}^{s} g(s, \tau, x(\tau), Q(\tau)) d \tau\right] d s\right\| \\
& \leq M_{1}\left(M_{2}+a M_{3}\right) \epsilon,
\end{aligned}
$$


which implies that $Y_{0}(t)$ is totally bounded, that is, $Y_{0}(t)$ is precompact in $X$. We will show that

$$
\Psi\left(Y_{0}\right)=S=\left\{(\Psi x): x \in Y_{0}\right\}
$$

is an equicontinuous family of functions.

For $0<t<s$, we have

$$
\begin{aligned}
\|(\Psi x)( & t)-(\Psi x)(s) \| \\
\leq & \left\|(R(t)-R(s)) x_{0}\right\|+\left\|(R(t)-R(s)) h\left(x\left(t_{1}\right), \ldots, x\left(t_{p}\right)\right)\right\| \\
& +\left\|\int_{0}^{t}(R(t-\tau)-R(s-\tau))\left[f(\tau, x(\tau))+\int_{0}^{\tau} g(\tau, \theta, x(\theta), Q(\theta)) d \theta\right] d \tau\right\| \\
& +\left\|\int_{t}^{s} T(s-\tau)\left[f(\tau, u(\tau))+\int_{0}^{\tau} g(\tau, \theta, x(\theta), Q(\theta)) d \theta\right] d \tau\right\| \\
\leq & \|R(t)-R(s)\|\left(\left\|x_{0}\right\|+H\right)+\left(M_{2}+a M_{3}\right) M_{1}|s-t| \\
& +\left(M_{2}+a M_{3}\right) \int_{0}^{t}\|R(t-\tau)-R(s-\tau)\| d \tau .
\end{aligned}
$$

The right-hand side of the above inequality is independent of $x \in Y_{0}$ and tends to zero as $s \rightarrow t$ (as a consequence of the continuity of $R(t)$ in the uniform operator topology for $t>0$ which follows from the compactness of $R(t), t>0)$. It is also clear that $S$ is bounded in $Y$. Thus by Arzelá-Ascoli's theorem, $S$ is precompact. Hence by the Schauder fixedpoint theorem, $\Psi$ has a fixed point in $Y_{0}$ and any fixed point of $\Psi$ is a mild solution of the nonlocal Cauchy problem (2.6).

Next we prove the strong solution of (2.6).

Theorem 3.2. Assume that conditions (i), (ii), (iii), and (iv) hold. Further, assume that

(v) $X$ is a reflexive Banach space,

(vi) $f: J \times X \rightarrow X$ is continuous in $t$ on $J$ and there exists a constant $M_{5}>0$ such that

$$
\|f(t, x)-f(s, y)\| \leq M_{5}(|t-s|+\|x-y\|) \quad t, s \in J, x, y \in X_{r}
$$

(vii) $g: \Delta \times X \times X \rightarrow X$ is continuous in $t$ on $J$ and there exists a constant $M_{6}>0$ such that

$$
\|g(t, \tau, x, y)-g(s, \tau, x, y)\| \leq M_{6}|t-s| \quad(t, s) \in \Delta, x, y \in X_{r}
$$

(viii) $x_{0} \in D(A), h\left(x\left(t_{1}\right), \ldots, x\left(t_{p}\right)\right) \in D(A)$.

(ix) $x$ is the unique mild solution of problem (2.6).

Then $x$ is the unique strong solution of problem (2.6). 
Proof. Since all the assumptions of Theorem 3.1 are satisfied, problem (2.6) possesses a mild solution $x$ which, according to assumption (ix), is the unique mild solution of problem (2.6). We will show that $x$ is a strong solution of problem (2.6) on J. For any $t \in J$, we have

$$
\begin{aligned}
x(t+\delta) & -x(t) \\
= & (R(t+\delta)-R(t))\left[x_{0}-h\left(x\left(t_{1}\right), \ldots, x\left(t_{p}\right)\right)\right] \\
& +\int_{0}^{\delta} R(t+\delta-s)\left[f(s, x(s))+\int_{0}^{s} g(s, \tau, x(\tau), Q(\tau)) d \tau\right] d s \\
& +\int_{\delta}^{t+\delta} R(t+\delta-s)\left[f(s, x(s))+\int_{0}^{s} g(s, \tau, x(\tau), Q(\tau)) d \tau\right] d s \\
& -\int_{0}^{t} R(t-s)\left[f(s, x(s))+\int_{0}^{s} g(s, \tau, x(\tau), Q(\tau)) d \tau\right] d s \\
= & (R(t+\delta)-R(t))\left[x_{0}-h\left(x\left(t_{1}\right), \ldots, x\left(t_{p}\right)\right)\right] \\
& +\int_{0}^{\delta} R(t+\delta-s)\left[f(s, x(s))+\int_{0}^{s} g(s, \tau, x(\tau), Q(\tau)) d \tau\right] d s \\
& +\int_{0}^{t} R(t-s)[f(s+\delta, x(s+\delta))-f(s, x(s))] d s \\
& +\int_{0}^{t} R(t-s) \int_{0}^{s}[g(s+\delta, \tau, x(\tau), Q(\tau))-g(s+\delta, \tau, x(\tau), Q(\tau)) d \tau] d s \\
& +\int_{0}^{t} R(t-s) \int_{0}^{s+\delta} g(s+\delta, \tau, x(\tau), Q(\tau)) d \tau d s .
\end{aligned}
$$

From our assumptions, we have

$$
\begin{aligned}
&\|x(t+\delta)-x(t)\| \\
& \leq\left\|[R(t+\delta)-R(t)]\left[x_{0}-h\left(x\left(t_{1}\right), \ldots, x\left(t_{p}\right)\right)\right]\right\|+\delta M_{1}\left(M_{2}+a M_{3}\right) \\
&+\int_{0}^{t} M_{1} M_{5}[\delta+\|x(s+\delta)-x(s)\|] d s+a M_{1} M_{6} \delta\left(a+M_{3}\right) \\
& \leq \delta\left(\left\|A x_{0}\right\|+\|A h\|\right)\left(M_{1}+a\|F\| M_{1}\right)+\delta M_{1}\left(M_{2}+a M_{3}\right) \\
&+M_{1} M_{5} \delta+a M_{1} M_{6} \delta\left(a+M_{3}\right)+M_{1} M_{5} \int_{0}^{t}\|x(s+\delta)-x(s)\| d s \\
&= P \delta+M_{1} M_{5} \int_{0}^{t}\|x(s+\delta)-x(s)\| d s,
\end{aligned}
$$

where

$$
P=\left(\left\|A x_{0}\right\|+\|A h\|\right)\left(M_{1}+a\|F\| M_{1}\right)+M_{1}\left(M_{2}+a M_{3}\right)+M_{1} M_{5}+a M_{1} M_{6}\left(a+M_{3}\right) .
$$

Using Gronwall's inequality, we get

$$
\|x(t+\delta)-x(t)\| \leq P \delta e^{a M_{1} M_{5}}, \quad t \in J .
$$

Therefore $x$ is Lipschitz continuous on $J$. 
The Lipschitz continuity of $x$ on $J$ combined with conditions (vi) and (vii) implies that $t \rightarrow f(t, x(t)), t \rightarrow g(t, s, x(t), Q(s))$ are Lipschitz continuous on $J$. By $[19,20]$ and the definition of strong solution, we see that the linear Cauchy problem

$$
\begin{gathered}
\frac{d v(t)}{d t}=A\left[v(t)+\int_{0}^{t} F(t-s) v(s) d s\right]+f(t, x(t))+\int_{0}^{t} g(t, s, x(s), Q(s)) d s, \quad t \in[0, a] \\
v(0)=x_{0}-h\left(x\left(t_{1}\right), \ldots, x\left(t_{p}\right)\right)
\end{gathered}
$$

has a unique strong solution $v$ satisfying the equation

$$
\begin{aligned}
v(t)= & R(t) x_{0}-R(t) h\left(x\left(t_{1}\right), \ldots, x\left(t_{p}\right)\right) \\
& +\int_{0}^{t} R(t-s)\left[f(s, x(s))+\int_{0}^{s} g(s, \tau, x(\tau), Q(\tau)) d \tau\right] d s \\
= & x(t) .
\end{aligned}
$$

Consequently $x$ is the unique strong solution of problem (2.6) on $J$.

\section{Controllability results}

In this section, we will study a new type of controllability problem for integrodifferential systems in Banach spaces. With the help of the fixed-point argument, several authors have investigated the problem of controllability of nonlinear systems in Banach spaces $[4,8$, $15,22,26,34,35]$. In particular, the Schauder fixed-point theorem is used to study the controllability of Volterra systems in $[27,30]$ and integrodifferential systems in $[2,5,32]$. Now we will establish a set of sufficient conditions for the controllability of semilinear integrodifferential system (4.1a) with nonlocal condition (4.1b).

Consider the following semilinear integrodifferential system with control parameter:

$$
\begin{gathered}
x^{\prime}(t)=A\left[x(t)+\int_{0}^{t} F(t-s) x(s) d s\right] \\
+(B u)(t)+f(t, x(t))+\int_{0}^{t} g(t, s, x(s), Q(s)) d s, \\
x(0)+h\left(x\left(t_{1}\right), \ldots, x\left(t_{p}\right)\right)=x_{0},
\end{gathered}
$$

where the state $x(\cdot)$ takes values in the Banach space $X$ and the control function $u(\cdot)$ is given in $L^{2}(J, U)$, a Banach space of admissible control functions with $U$ as a Banach space. Here $B$ is a bounded linear operator from $U$ into $X$. Take $J=[0, T]$. Then, for system (4.1a), there exists a mild solution of the following form:

$$
\begin{aligned}
x(t)= & R(t)\left[x_{0}-h\left(x\left(t_{1}\right), \ldots, x\left(t_{p}\right)\right)\right] \\
& +\int_{0}^{t} R(t-s)\left[(B u)(s)+f(s, x(s))+\int_{0}^{s} g(s, \tau, x(\tau), Q(\tau)) d \tau\right] d s,
\end{aligned}
$$


where the resolvent operator $R(t) \in B(X)$ for $t \in J$ and the functions $f, g, k$, and $h$ satisfy the conditions stated in Section 3.

Definition 4.1. System (4.1a) is said to be controllable with nonlocal condition (4.1b) on the interval $J$ if , for every $x_{0}, x_{T} \in X$, there exists a control $u \in L^{2}(J, U)$ such that the mild solution $x(\cdot)$ of $(4.1 \mathrm{a})$ satisfies

$$
x(0)+h\left(x\left(t_{1}\right), \ldots, x\left(t_{p}\right)\right)=x_{0}, \quad x(T)=x_{T} .
$$

To establish the result, we need the following additional hypothesis.

(x) The linear operator $W$ from $U$ into $X$, defined by

$$
W u=\int_{0}^{T} R(T-s) B u(s) d s,
$$

induces an inverse operator $\tilde{W}^{-1}$ defined on $L^{2}(J ; U) / \operatorname{ker} W$, and there exists a constant $M_{4}>0$ such that $\left\|B \tilde{W}^{-1}\right\| \leq M_{4}$.

Theorem 4.2. If hypotheses (i), (ii), (iii), (iv), and (x) are satisfied, then system (4.1) is controllable on $\mathrm{J}$.

Proof. Using hypothesis $(\mathrm{x})$, for an arbitrary function $x(\cdot)$, define the control

$$
\begin{aligned}
u(t)=\tilde{W}^{-1}[ & x_{T}-R(T)\left[x_{0}-h\left(x\left(t_{1}\right), \ldots, x\left(t_{p}\right)\right)\right] \\
& \left.\quad-\int_{0}^{T} R(T-s)\left[f(s, x(s))+\int_{0}^{s} g(s, \tau, x(\tau), Q(\tau)) d \tau\right] d s\right](t) .
\end{aligned}
$$

Now we will show that, when using this control, the operator, defined by

$$
\begin{aligned}
(\Phi x)(t)= & R(t)\left[x_{0}-h\left(x\left(t_{1}\right), \ldots, x\left(t_{p}\right)\right)\right] \\
& +\int_{0}^{t} R(t-s)\left[(B u)(s)+f(s, x(s))+\int_{0}^{s} g(s, \tau, x(\tau), Q(\tau)) d \tau\right] d s
\end{aligned}
$$

has a fixed point. This fixed point is then a solution of (4.1a).

Clearly, $(\Phi x)(T)=x_{T}$, which means that the control $u$ steers the semilinear integrodifferential system from the initial state $x_{0}$ to $x_{T}$ in time $T$ provided we can obtain a fixed point of the nonlinear operator $\Phi$. Let

$$
Z_{0}=\left\{x \in Y: x(0)+h\left(x\left(t_{1}\right), \ldots, x\left(t_{p}\right)\right)=x_{0},\|x(t)\| \leq r, \text { for } t \in J\right\},
$$


74 Existence of solutions and controllability

where the positive constant $r$ is given by

$$
\begin{aligned}
r= & M_{1}\left(\left\|x_{0}\right\|+H\right) \\
& +T M_{1} M_{4}\left[\left\|x_{T}\right\|+M_{1}\left(\left\|x_{0}\right\|+H\right)+T M_{1}\left(M_{2}+M_{3} T\right)\right] \\
& +T M_{1}\left(M_{2}+M_{3} T\right)
\end{aligned}
$$

Then $Z_{0}$ is clearly a bounded, closed, and convex subset of $Z$. Define a mapping $\Phi: Z \rightarrow$ $Z_{0}$ by

$$
\begin{aligned}
&(\Phi x)(t)= R(t)\left[x_{0}-h\left(x\left(t_{1}\right), \ldots, x\left(t_{p}\right)\right)\right] \\
&+\int_{0}^{t} R(t-\eta) B \tilde{W}^{-1} \\
& \times\left[x_{T}-R(T)\left[x_{0}-h\left(x\left(t_{1}\right), \ldots, x\left(t_{p}\right)\right)\right]\right. \\
&\left.\quad \quad-\int_{0}^{T} R(T-s)\left[f(s, x(s))+\int_{0}^{s} g(s, \tau, x(\tau), Q(\tau)) d \tau\right] d s\right](\theta) d \theta \\
&+\int_{0}^{t} R(t-s)\left[f(s, x(s))+\int_{0}^{s} g(s, \tau, x(\tau), Q(\tau)) d \tau\right] d s .
\end{aligned}
$$

Since $f$ and $g$ are continuous and $\|(\Phi x)(t)\| \leq r$, it follows that $\Phi$ is continuous and maps $Z_{0}$ into itself. Moreover, $\Phi$ maps $Z_{0}$ into a precompact subset of $Z_{0}$. To prove this, we first show that for every fixed $t \in J$, the set

$$
Z_{0}(t)=\left\{(\Phi x)(t): x \in Z_{0}\right\}
$$

is precompact in $X$. This is clear for $t=0$ since $Z_{0}(0)$ is precompact by assumption (iv).

Let $t>0$ be fixed and for $0<\epsilon<t$, define

$$
\begin{aligned}
\left(\Phi_{\epsilon} x\right)(t)= & R(t)\left[x_{0}-h\left(x\left(t_{1}\right), \ldots, x\left(t_{p}\right)\right)\right] \\
+ & \int_{0}^{t-\epsilon} R(t-\eta) B \tilde{W}^{-1} \\
& \times\left[x_{T}-R(T)\left[x_{0}-h\left(x\left(t_{1}\right), \ldots, x\left(t_{p}\right)\right)\right]\right. \\
& \left.\quad-\int_{0}^{T} R(T-s)\left[f(s, x(s))+\int_{0}^{s} g(s, \tau, x(\tau), Q(\tau)) d \tau\right] d s\right](\theta) d \theta \\
+ & \int_{0}^{t-\epsilon} R(t-s)\left[f(s, x(s))+\int_{0}^{s} g(s, \tau, x(\tau), Q(\tau)) d \tau\right] d s .
\end{aligned}
$$

Since $R(t)$ is compact for every $t>0$, the set

$$
Z_{\epsilon}(t)=\left\{\left(\Phi_{\epsilon} x\right)(t): x \in Z_{0}\right\}
$$


is precompact in $X$ for every $\epsilon, 0<\epsilon<t$. Furthermore, for $x \in Z_{0}$, we have

$$
\begin{aligned}
& \left\|(\Phi x)(t)-\left(\Phi_{\epsilon} x\right)(t)\right\| \\
& \leq \| \int_{t-\epsilon}^{t} R(t-\eta) B \tilde{W}^{-1} \\
& \quad \times\left[x_{T}-R(T)\left[x_{0}-h\left(x\left(t_{1}\right), \ldots, x\left(t_{p}\right)\right)\right]\right. \\
& \left.\quad-\int_{0}^{T} R(T-s)\left[f(s, x(s))+\int_{0}^{s} g(s, \tau, x(\tau), Q(\tau)) d \tau\right] d s\right](\theta) d \theta \| \\
& +\left\|\int_{t-\epsilon}^{t} R(t-s)\left[f(s, x(s))+\int_{0}^{s} g(s, \tau, x(\tau), Q(\tau)) d \tau\right] d s\right\| \\
& \leq \epsilon M_{1} M_{4}\left[\left\|x_{T}\right\|+M_{1}\left(\left\|x_{0}\right\|+H\right)+M_{1}\left(M_{2}+M_{3} T\right) T\right]+\epsilon M_{1}\left[M_{2}+M_{3} T\right],
\end{aligned}
$$

which implies that $Z_{0}(t)$ is totally bounded, that is, precompact in $X$. We want to show that

$$
\Phi\left(Z_{0}\right)=\left\{\Phi x: x \in Z_{0}\right\}
$$

is an equicontinuous family of functions. For that, let $t_{2}>t_{1}>0$. Then, we have

$$
\begin{aligned}
& \left\|(\Phi x)\left(t_{1}\right)-(\Phi x)\left(t_{2}\right)\right\| \\
& \leq\left\|R\left(t_{1}\right)-R\left(t_{2}\right)\right\|\left(\left\|x_{0}\right\|+H\right) \\
& +\| \int_{0}^{t_{1}}\left[R\left(t_{1}-\theta\right)-R\left(t_{2}-\theta\right)\right] B \tilde{W}^{-1} \\
& \quad \times\left[x_{T}-R(T)\left[x_{0}-h\left(x\left(t_{1}\right), \ldots, x\left(t_{p}\right)\right)\right]\right. \\
& \left.\quad-\int_{0}^{T} R(T-s)\left[f(s, x(s))+\int_{0}^{s} g(s, \tau, x(\tau), Q(\tau)) d \tau\right] d s\right](\theta) d \theta \\
& -\int_{t_{1}}^{t_{2}} R\left(t_{2}-\eta\right) B \tilde{W}^{-1} \\
& \quad \times\left[x_{T}-R(T)\left[x_{0}-h\left(x\left(t_{1}\right), \ldots, x\left(t_{p}\right)\right)\right]\right. \\
& +\| \int_{0}^{t_{1}}\left[R\left(t_{1}-s\right)-R\left(t_{2}-s\right)\right]\left[f(s, x(s))+\int_{0}^{s} g(s, \tau, x(\tau), Q(\tau)) d \tau\right] d s \\
& \quad-\int_{t_{1}}^{t_{2}} R\left(t_{2}-s\right)\left[f(s, x(s))+\int_{0}^{s} g(s, \tau, x(\tau), Q(\tau)) d \tau\right] d s \|
\end{aligned}
$$




$$
\begin{aligned}
\leq & \left\|R\left(t_{1}\right)-R\left(t_{2}\right)\right\|\left(\left\|x_{0}\right\|+H\right) \\
& +\int_{0}^{t_{1}}\left\|R\left(t_{1}-s\right)-R\left(t_{2}-s\right)\right\| M_{4}\left[\left\|x_{T}\right\|+M_{1}\left(\left\|x_{0}\right\|+H\right)+M_{1}\left(M_{2}+M_{3} T\right) T\right] d s \\
& +\int_{t_{1}}^{t_{2}}\left\|R\left(t_{2}-s\right)\right\| M_{4}\left[\left\|x_{T}\right\|+M_{1}\left(\left\|x_{0}\right\|+H\right)+M_{1}\left(M_{2}+M_{3} T\right) T\right] d s \\
& +\int_{0}^{t_{1}}\left\|R\left(t_{1}-s\right)-R\left(t_{2}-s\right)\right\|\left(M_{2}+M_{3} T\right) d s \\
& +\int_{t_{1}}^{t_{2}}\left\|R\left(t_{2}-s\right)\right\|\left(M_{2}+M_{3} T\right) d s .
\end{aligned}
$$

The compactness of $R(t), t>0$, implies that $R(t)$ is continuous in the uniform operator topology for $t>0$. Thus, the right-hand side of (4.15), which is independent of $x \in Z_{0}$, tends to zero as $t_{2}-t_{1} \rightarrow 0$. So $\Phi\left(Z_{0}\right)$ is an equicontinuous family of functions.

Also $\Phi\left(Z_{0}\right)$ is bounded in $Z$, and so by the Arzelá-Ascoli theorem, $\Phi\left(Z_{0}\right)$ is precompact. Hence, from the Schauder fixed-point theorem, $\Phi$ has a fixed point in $Z_{0}$. Any fixed point of $\Phi$ is a mild solution of (4.1a) on $J$ satisfying $(\Phi x)(t)=x(t) \in X$. Thus, system (4.1a) is controllable on $J$.

\section{Examples}

We give some examples of nonlocal conditions. Let $p \in N$ and let $t_{1}, \ldots, t_{p}$ be given real numbers such that $0<t_{1}<\cdots<t_{p} \leq a$. Theorems 3.1 and 3.2 can be applied for $h$ defined by the formula

$$
h(y)=\sum_{i=0}^{p} c_{i} y\left(t_{i}\right) \quad \text { for } y \in X
$$

or

$$
h(y)=\sum_{i=0}^{p} \frac{c_{i}}{\epsilon_{i}} \int_{t_{i}-\epsilon_{i}}^{t_{i}} y(s) d s \quad \text { for } y \in X,
$$

where $c_{i}, \epsilon_{i}(i=1, \ldots, p)$ are given positive constants such that $0<t_{1}-\epsilon_{1}$ and $t_{i-1}<t_{i}-\epsilon_{i}$ $(i=1, \ldots, p)$. For more examples on various types of nonlocal conditions, one can refer to $[10,11,14,25]$.

Consider the following simplified classical heat equation for material with memory:

$$
\begin{aligned}
z_{t}(t, x)= & \frac{\partial^{2}}{\partial x^{2}}\left[z(t, x)+\int_{0}^{t} b(t-s) z(s, x) d s\right]+B u(t) \\
& +p(t, z(t, x))+\int_{0}^{t} q\left(t, s, z(s, x), \int_{0}^{s} e(s, \tau, z(\tau, x)) d \tau\right) d s
\end{aligned}
$$


and the given nonlocal initial and boundary conditions:

$$
\begin{gathered}
z(0, t)=z(1, t)=0, \quad x \in I=(0,1), \quad t \in J, \\
z(x, 0)+h(z(x, \cdot))=z_{0}(x),
\end{gathered}
$$

where $b$ is continuous and bounded and $h$ satisfies an appropriate condition. Here $B: U \rightarrow$ $X$ is a linear operator such that there exists an inverse operator $\tilde{W}^{-1}$ on $L^{2}(J ; U) / \operatorname{ker} W$, where $W$ is defined by

$$
W u=\int_{0}^{T} R(T-s) B u(s) d s
$$

The resolvent operator $R(t)$ is compact [28] and $p: J \times X \rightarrow X, e: \Delta \times X \rightarrow X$, and $q$ : $\Delta \times X \times X \rightarrow X$ are all continuous and uniformly bounded. Problem (5.3) can be brought to the form of (4.1a) by making suitable choices of $A, B, f, k$, and $g$ as follows.

Let $X=U=L^{2}(J, R), A w=w_{x x}$, and $D(A)=\left\{w \in X: w_{x x} \in X, w(0)=w(1)=0\right\}$. Let

$$
\begin{gathered}
f(t, w)(x)=p(t, w(x)), \quad(t, w) \in J \times X, \\
k(t, s, w)(x)=e(t, s, w(x)), \\
g(t, s, w, \sigma)(x)=q(t, s, w(x), \sigma(x)), \quad x \in I,
\end{gathered}
$$

be such that condition (ii) is satisfied. Then system (5.3) becomes an abstract formulation of (4.1a). Also by [24, Theorem 3] the solutions are all bounded. Further, all the conditions stated in the above theorem are satisfied. Hence system (5.3) is controllable on $J$.

\section{Acknowledgment}

The work of the first author was supported by KOSEF during 1999-2000.

\section{References}

[1] K. Balachandran, Existence and uniqueness of mild and strong solutions of nonlinear integrodifferential equations with nonlocal condition, Differential Equations Dynam. Systems 6 (1998), no. 1-2, 159-165.

[2] K. Balachandran, P. Balasubramaniam, and J. P. Dauer, Local null controllability of nonlinear functional-differential systems in Banach space, J. Optim. Theory Appl. 88 (1996), no. 1, 6175.

[3] K. Balachandran and M. Chandrasekaran, Nonlocal Cauchy problem for quasilinear integrodifferential equation in Banach spaces, Dynam. Systems Appl. 8 (1999), no. 1, 35-43.

[4] K. Balachandran and J. P. Dauer, Local controllability of semilinear evolution systems in Banach spaces, Indian J. Pure Appl. Math. 29 (1998), no. 3, 311-320.

[5] K. Balachandran, J. P. Dauer, and P. Balasubramaniam, Controllability of nonlinear integrodifferential systems in Banach space, J. Optim. Theory Appl. 84 (1995), no. 1, 83-91.

[6] K. Balachandran and S. Ilamaran, Existence and uniqueness of mild and strong solutions of a Volterra integrodifferential equation with nonlocal conditions, Tamkang J. Math. 28 (1997), no. 2, 93-100. 
[7] K. Balachandran, D. G. Park, and Y. C. Kwun, Nonlinear integrodifferential equations of Sobolev type with nonlocal conditions in Banach spaces, Commun. Korean Math. Soc. 14 (1999), no. $1,223-231$.

[8] W. M. Bian, Controllability of nonlinear evolution systems with preassigned responses, J. Optim. Theory Appl. 100 (1999), no. 2, 265-285.

[9] L. Byszewski, Theorems about the existence and uniqueness of solutions of a semilinear evolution nonlocal Cauchy problem, J. Math. Anal. Appl. 162 (1991), no. 2, 494-505.

[10] Existence of approximate solution to abstract nonlocal Cauchy problem, J. Appl. Math. Stochastic Anal. 5 (1992), no. 4, 363-373.

[11] On weak solutions of functional-differential abstract nonlocal Cauchy problems, Ann. Polon. Math. 65 (1997), no. 2, 163-170.

[12] _ Existence and uniqueness of a classical solution to a functional-differential abstract nonlocal Cauchy problem, J. Appl. Math. Stochastic Anal. 12 (1999), no. 1, 91-97.

[13] L. Byszewski and H. Akca, On a mild solution of a semilinear functional-differential evolution nonlocal problem, J. Appl. Math. Stochastic Anal. 10 (1997), no. 3, 265-271.

[14] Existence of solutions of a semilinear functional-differential evolution nonlocal problem, Nonlinear Anal. 34 (1998), no. 1, 65-72.

[15] E. N. Chukwu and S. M. Lenhart, Controllability questions for nonlinear systems in abstract spaces, J. Optim. Theory Appl. 68 (1991), no. 3, 437-462.

[16] C. Corduneanu, Integral Equations and Applications, Cambridge University Press, Cambridge, 1991.

[17] J. P. Dauer and K. Balachandran, Existence of solutions for an integrodifferential equation with nonlocal condition in Banach spaces, Libertas Math. 16 (1996), 133-143.

[18] K. Deng, Exponential decay of solutions of semilinear parabolic equations with nonlocal initial conditions, J. Math. Anal. Appl. 179 (1993), no. 2, 630-637.

[19] W. Desch, R. Grimmer, and W. Schappacher, Well-posedness and wave propagation for a class of integrodifferential equations in Banach space, J. Differential Equations 74 (1988), no. 2, 391-411.

[20] R. C. Grimmer, Resolvent operators for integral equations in a Banach space, Trans. Amer. Math. Soc. 273 (1982), no. 1, 333-349.

[21] G. Gripenberg, S.-O. Londen, and O. Staffans, Volterra Integral and Functional Equations, Encyclopedia of Mathematics and Its Applications, vol. 34, Cambridge University Press, Cambridge, 1990.

[22] H.-K. Han and J.-Y. Park, Boundary controllability of differential equations with nonlocal condition, J. Math. Anal. Appl. 230 (1999), no. 1, 242-250.

[23] M. L. Heard and S. M. Rankin, Nonlinear Volterra integrodifferential equations, J. Math. Anal. Appl. 188 (1994), no. 2, 569-589.

[24] M. A. Hussain, On a nonlinear integrodifferential equation in Banach space, Indian J. Pure Appl. Math. 19 (1988), no. 6, 516-529.

[25] D. Jackson, Existence and uniqueness of solutions to semilinear nonlocal parabolic equations, J. Math. Anal. Appl. 172 (1993), no. 1, 256-265.

[26] J. Klamka, Constrained controllability of nonlinear systems, J. Math. Anal. Appl. 201 (1996), no. 2, 365-374.

[27] Y. C. Kwun, J. Y. Park, and J. W. Ryu, Approximate controllability and controllability for delay Volterra system, Bull. Korean Math. Soc. 28 (1991), no. 2, 131-145.

[28] Y. P. Lin and J. H. Liu, Semilinear integrodifferential equations with nonlocal Cauchy problem, Nonlinear Anal. 26 (1996), no. 5, 1023-1033.

[29] R. C. MacCamy, An integro-differential equation with application in heat flow, Quart. Appl. Math. 35 (1977), no. 1, 1-19. 
[30] K. Naito, On controllability for a nonlinear Volterra equation, Nonlinear Anal. 18 (1992), no. 1, 99-108.

[31] S. K. Ntouyas and P. Ch. Tsamatos, Global existence for semilinear evolution equations with nonlocal conditions, J. Math. Anal. Appl. 210 (1997), no. 2, 679-687.

[32] J. Y. Park and H. K. Han, Controllability of nonlinear functional integro-differential systems in Banach space, Nihonkai Math. J. 8 (1997), no. 1, 47-53.

[33] A. Pazy, Semigroups of Linear Operators and Applications to Partial Differential Equations, Applied Mathematical Sciences, vol. 44, Springer-Verlag, New York, 1983.

[34] M. D. Quinn and N. Carmichael, An approach to nonlinear control problems using fixed-point methods, degree theory and pseudo-inverses, Numer. Funct. Anal. Optim. 7 (1984/1985), no. 2-3, 197-219.

[35] M. Yamamoto and J. Y. Park, Controllability for parabolic equations with uniformly bounded nonlinear terms, J. Optim. Theory Appl. 66 (1990), no. 3, 515-532.

[36] J. A. Yorke, A continuous differential equation in Hilbert space without existence, Funkcial. Ekvac. 13 (1970), 19-21.

K. Balachandran: Department of Mathematics, Bharathiar University, Coimbatore 641 046, India E-mail address: balachandran_k@lycos.com

J. Y. Park: Department of Mathematics, Pusan National University, Pusan 609-735, Korea

E-mail address: jyepark@pusan.ac.kr 


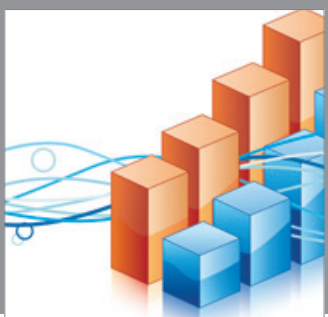

Advances in

Operations Research

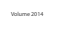

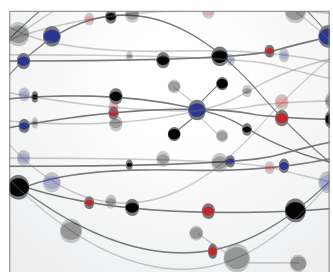

\section{The Scientific} World Journal
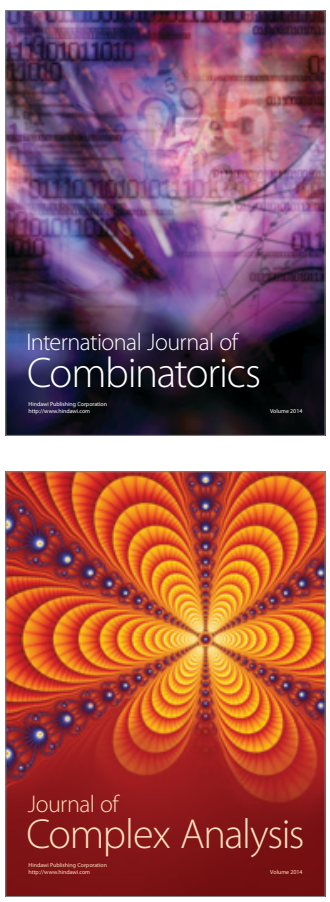

International Journal of

Mathematics and

Mathematical

Sciences
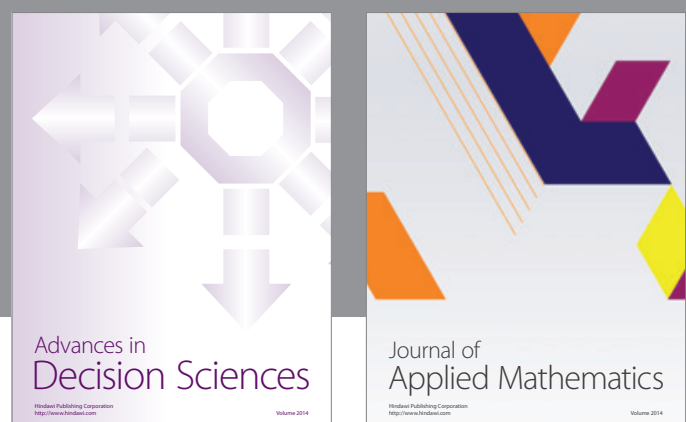

Journal of

Applied Mathematics
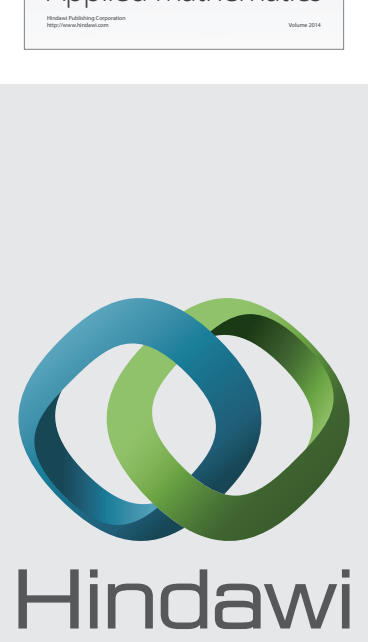

Submit your manuscripts at http://www.hindawi.com
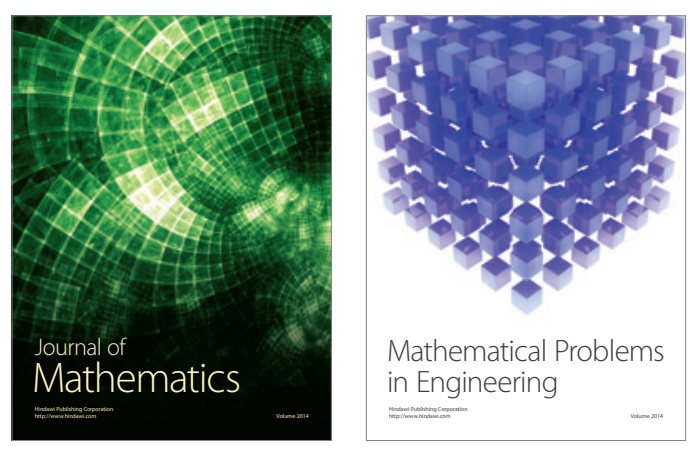

Mathematical Problems in Engineering
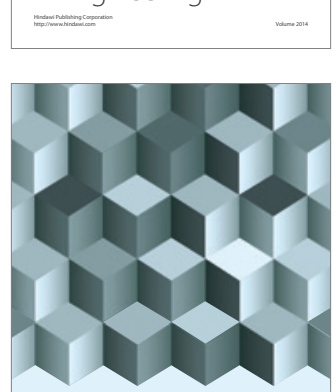

Journal of

Function Spaces
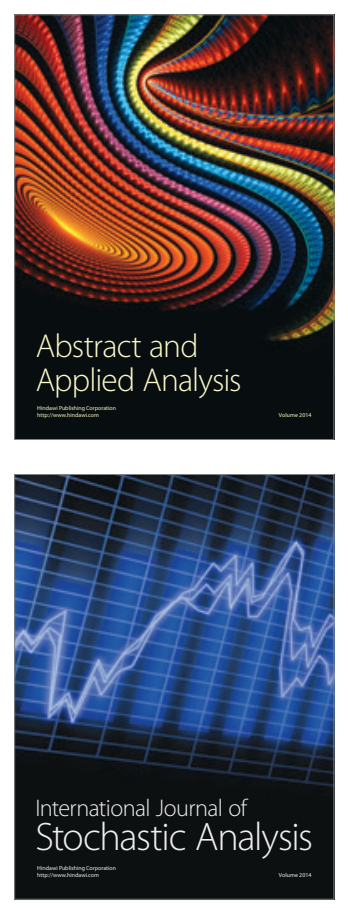

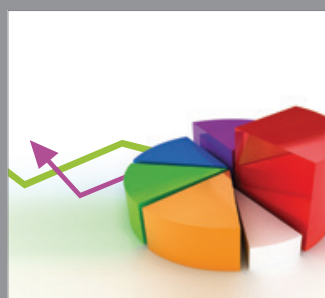

ournal of

Probability and Statistics

Promensencen
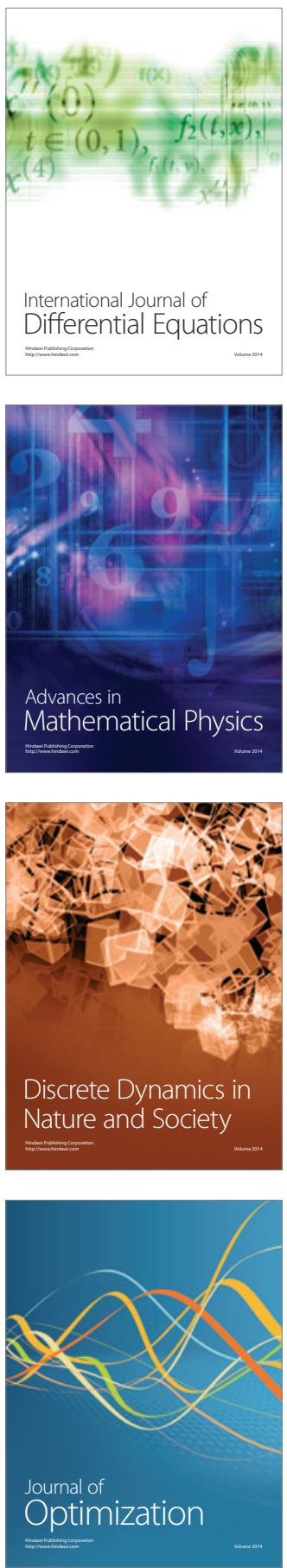